\title{
Mach-Zehnder interferometer by utilizing phase modulation of transmitted light through magnetic fluid films possessing tunable refractive index
}

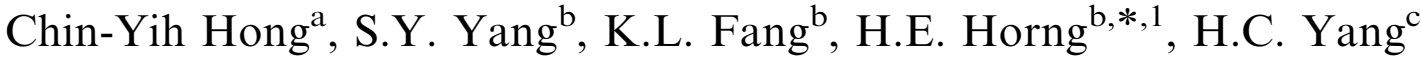 \\ ${ }^{a}$ Department of Mechanical and Automation Engineering, Da-Yeh University, Chang-Hwa 515, Taiwan \\ ${ }^{\mathrm{b}}$ Institute of Electro-optical Science and Technology, National Taiwan Normal University, Taipei 116, Taiwan \\ ${ }^{\mathrm{c}}$ Department of Physics, National Taiwan University, Taipei 106, Taiwan
}

Received 23 September 2004; received in revised form 15 February 2005

Available online 11 March 2005

\begin{abstract}
Due to its diverse applications in photonics, bio-sensors, mechanics, etc., Mach-Zehnder interferometer becomes one of important devices. Hence, lots of efforts have been paid to develop advanced Mach-Zehnder interferometers. In this work, we explore new-model Mach-Zehnder interferometer, in which one of arm is consisted of magnetic fluid films. By utilizing the tunable refractive index of magnetic fluid films under external magnetic fields, the traveling phase of a propagating light through the magnetic fluid film is changed. This could lead to a variation in the interfered intensity of the Mach-Zehnder interferometer when an external magnetic field is applied. The modulation in the interfered intensity by the external magnetic field is demonstrated experimentally, and the relevant physical origin is also discussed.

(C) 2005 Elsevier B.V. All rights reserved.
\end{abstract}

PACS: $85.60 . \mathrm{Bt} ; 75.50 . \mathrm{Mm} ; 78.20 . \mathrm{Ci}$

Keywords: Interferometer; Magnetic fluids

\footnotetext{
*Corresponding authors. Institute of Electro-optical Science and Technology, National Taiwan Normal University, 88, Sec. 4, Ting-Chou Rd., Taipei 116, Taiwan. Tel.: + 886229338260 ; fax: +886286631954 .

E-mail address: phyfv001@scc.ntnu.edu.tw (H.E. Horng).

${ }^{1}$ Also with Department of Physics, National Taiwan Normal University, Taipei 116, Taiwan.
}

\section{Introduction}

Mach-Zehnder interferometers are a kind of very important device because of its diverse applications in photonics [1,2], biosensors [3], mechanical sensors [4], etc. Hence, lots of scientists' and engineers' efforts are paid to develop Mach-Zehnder interferometers. The key architecture of a Mach-Zehnder interferometer is the two 
jointed arms, which show different traveling phase when two coherent lights propagate through these two arms. As a result, the intensity of the interference light can be modulated by varying the optical phase difference of these two arms. Usually, the variation in the optical phase difference is achieved by changing the refractive index of one arm. The control mechanism depends on the material and its physical intuition of tunable refractive index. For example, by heating one of the $\mathrm{Si}$ arms [5,6], the refractive index of this arm is changed to manipulate the phase difference. If the arms are made of electro-optic polymers or liquid crystals, the modulation in the refractive index of one arm can be achieved by applying an external electric field $[7,8]$. Another method is using photochromic polymers as one arm, which refractive index is reversibly changed under irradiation of lights with various wavelengths [9]. According to most published results, the refractive index of one arm of a Mach-Zehnder interferometer is adjusted via thermal, electric, or optical control. In this work, an alternative way to tune the refractive index of one arm is explored.

\section{Experimental details}

In our designed Mach-Zehnder interferometer, the light emitted from a $\mathrm{He}-\mathrm{Ne}$ laser is split into two coherent lights. One of the coherent lights travels in air (referred as path (1)), whereas the other transmits through a magnetic fluid film (referred as path (2)), as shown in Fig. 1. Then, these two coherent lights interfere with each. A typical interference pattern is also shown in Fig. 1. A photo-detector is posited at the center of the interference pattern to measure the intensity.

The magnetic fluid film consists of a glass cell containing water-based lauric-acid coated $\mathrm{Fe}_{3} \mathrm{O}_{4}$ magnetic fluid. The glass cell is $3 \times 3 \mathrm{~mm}^{2}$ in area and $260 \mu \mathrm{m}$ in depth. The magnetic fluid is fabricated via chemical co-precipitation method. The flow chart to illustrate the synthesis of magnetic fluids is shown in Fig. 2. The distribution in the diameter of $\mathrm{Fe}_{3} \mathrm{O}_{4}$ particles are measured through dynamic laser scattering technologies. Fig. 3 plots the diameter distribution of the

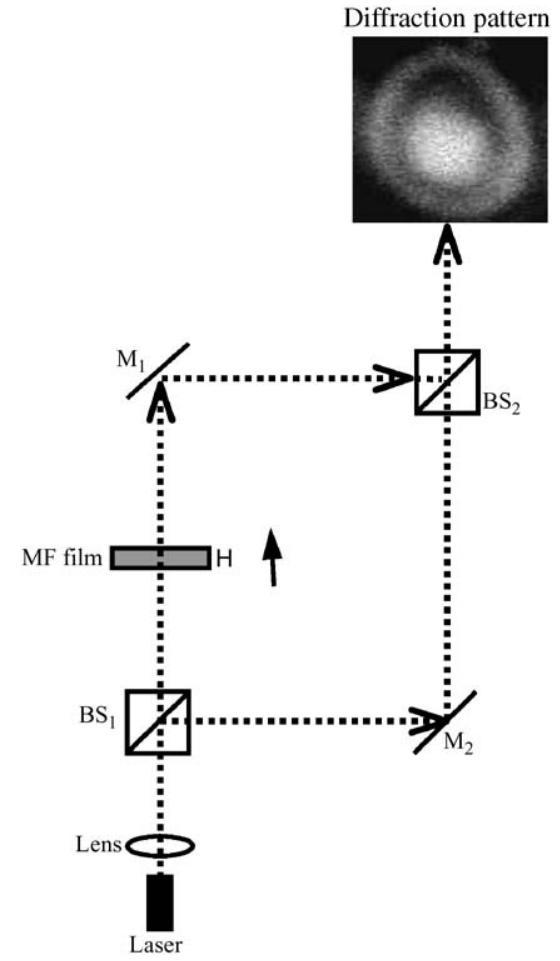

Fig. 1. Scheme of Mach-Zehnder interferometer. A magnetic fluid film is located in one of the arms of the interferometer. The light is normally incident into the film and an external magnetic field is applied along light propagation. $\mathrm{BS}_{1}$ and $\mathrm{BS}_{2}$ denote beam splitters, $\mathrm{M}_{1}$ and $\mathrm{M}_{2}$ are mirrors.

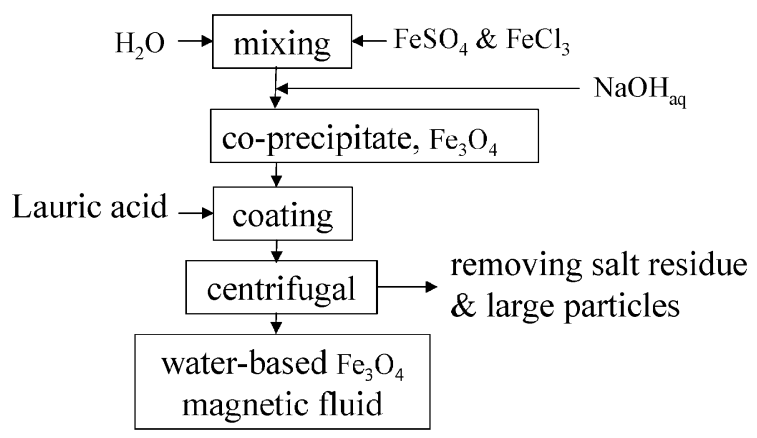

Fig. 2. Flow chart for the synthesis of lauric-acid coated $\mathrm{Fe}_{3} \mathrm{O}_{4}$ water-based magnetic fluid. The lauric acid is coated on $\mathrm{Fe}_{3} \mathrm{O}_{4}$ particles as a surfactant to make the particles dispersible in water.

$\mathrm{Fe}_{3} \mathrm{O}_{4}$ particles used here. The mean diameter and its standard deviation are 63.9 and $14.0 \mathrm{~nm}$, respectively. The magnetic fluid film is located in a 


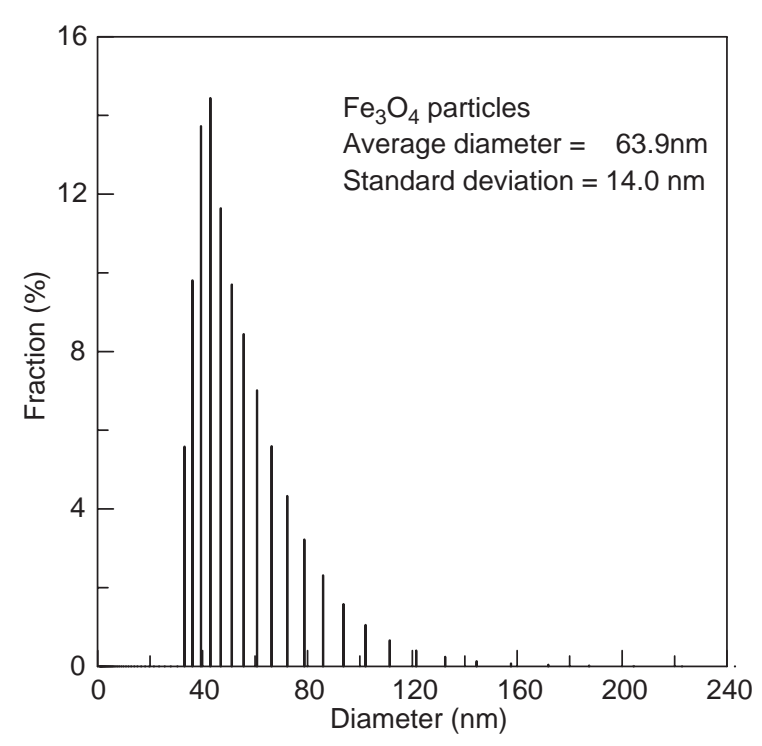

Fig. 3. Diameter distribution of $\mathrm{Fe}_{3} \mathrm{O}_{4}$ magnetic particles dispersed in water. The mean diameter and its standard deviation are 63.9 and $14.0 \mathrm{~nm}$, respectively.

solenoid which provides a magnetic field perpendicular to the film surface and parallel to the light propagation.

\section{Results and discussion}

It was reported that the refractive index of magnetic fluid is magnetic-field dependent; the higher the field, the larger the refractive index [10]. This implies that when the external magnetic field is adjusted, the traveling phase of the transmitted light through the magnetic fluid film deviates from that under zero field. Consequently, the interfered intensity detected by the photo-detector may be varied. To demonstrate this, the interfered intensity is measured for the case of a $260 \mu \mathrm{m}$-thick and $1.29 \mathrm{emu} / \mathrm{g}$-concentrated magnetic fluid film. The temporal variation in the applied magnetic field is shown in Fig. 4(a), and the corresponding dynamic response of the interfered intensity is shown in Fig. 4(c) with a solid line. It was found that the interfered intensity during the time interval at which the magnetic field is active becomes higher.

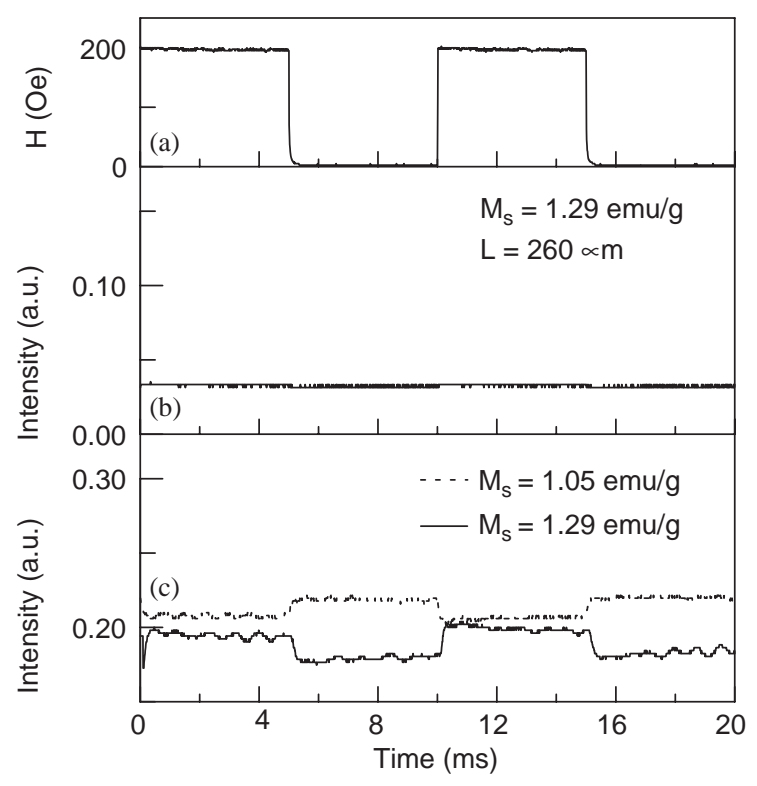

Fig. 4. Temporal variations of (a) applied magnetic field, and the corresponding (b) transmitted intensity through the magnetic fluid film and (c) interfered intensity of the Mach-Zehnder interferometer. The thickness $L$ and the concentration $M_{\mathrm{s}}$ of the magnetic fluid film investigated in (b) are $260 \mu \mathrm{m}$ and $1.29 \mathrm{emu} / \mathrm{g}$. The $260 \mu \mathrm{m}$-thickness magnetic fluid films of 1.05 and $1.29 \mathrm{emu} / \mathrm{g}$ in concentrations are used in (c).

For the interference of two coherent lights, the time-average interfered intensity is written as

$I=I_{1}+I_{2}+2 \sqrt{I_{1} I_{2}} \cos \phi$,

where $I_{1}$ and $I_{2}$ are the intensities of the two interfered lights propagating along path 1 and path 2 (through magnetic fluid film) respectively, and $\phi$ is the phase difference of the two lights. The phase difference $\phi$ can be written as

$\phi=\phi_{0}+2 \pi \lambda \Delta n / L$,

where $\phi_{0}$ is attributed to the asymmetric traveling lengths between path 1 and path 2 under zero magnetic field, $\lambda$ is the wavelength of the incident light ( $=632.8 \mathrm{~nm}$ here), $L$ is the traveling length through the magnetic fluid film, and $\Delta n$ denotes the variation in the refractive index of the magnetic fluid film under external magnetic fields with respect to the zero-field refractive index. It is clear that $\phi$ could be changed when a magnetic 
field is applied to the magnetic fluid film because the light along path 2 experiences a different refractive index $\Delta n$ through the film as compared to that under zero field. This would lead to a variation in the interfered intensity under an external magnetic field, as shown in Fig. 4(c). In addition to the change in the phase difference, the intensity $I_{2}$ may be varied when the refractive index of the magnetic fluid film varies under an external magnetic field. To clarify either $I_{2}$ or $\phi$ is dominant to the magnetic-field induced variation in the interfered intensity shown in Fig. 4(c), the corresponding dynamic response of the light intensity $I_{2}$ is detected and shown in Fig. 4(b). It was found that the transmitted intensity through the magnetic fluid film remains unchanged under the action of magnetic fields. This implies that the variation in the interfered intensity shown in Fig. 4(c) is attributed to the change in the traveling phase, not the intensity, of the transmitted light through the magnetic fluid when the magnetic field is adjusted.

In Eq. (1), the phase difference $\phi$ is crucial to the field-modulated behavior in the interfered intensity. Furthermore, in Eq. (2), the $\phi$ is the sum of $\phi_{0}$ and $2 \pi \lambda \Delta n / L$, where $\phi_{0}$ denotes the off-set part and $2 \pi \lambda \Delta n / L$ corresponds to the variation part. The $\phi_{0}$ is determined by the concentration of magnetic fluid, the film thickness and the phase difference resulted from the path difference in air between path 1 and path 2 , and the $\Delta n$ depends on the fluid concentration, film thickness, and field strength [11]. Hence, by selecting a suitable fluid concentration and the film thickness, the $\phi$ can be manipulated along the trend from zero toward $\pi$ when an external magnetic field is applied to the magnetic fluid film. Thus, the interfered intensity is reduced under the action of a magnetic field, as shown with the solid line in Fig. 4(c). On the other hand, the $\phi$ can be changed from $\pi$ toward $2 \pi$ under an external magnetic field by using a different concentration of magnetic fluid, which may exhibit an increase in the interfered intensity increases when a magnetic field is activated. This is evidenced with the dashed line shown in Fig. 4(c) by using the magnetic fluid with the concentration of $1.05 \mathrm{emu} / \mathrm{g}$. Therefore, the interfered intensity could be reduced or increased under the applica- tion of an external magnetic field, depending on how the phase difference changes. In addition, the field-induced variations in the interfered intensity, which is due to the $2 \pi \lambda \Delta n / L$, are 0.0216 and 0.0137 for the $1.29-\mathrm{emu} / \mathrm{g}$ and the $1.05-\mathrm{emu} / \mathrm{g}$ magnetic fluid films, respectively. A larger variation is achieved for a more concentrated magnetic fluid film. This is resulted from the larger increase in the refractive index $\Delta n$ of a more concentrated magnetic fluid film under a given magnetic field [11].

\section{Conclusion}

By utilizing the magnetically tunable refractive index of magnetic fluid films as one arm, a Mach-Zehnder interferometer is designed and its feasibility is demonstrated. The modulation of the interfered intensity was observed when an external magnetic field was applied to the magnetic fluid film. The origin account for this intensity modulation is attributed to the manipulation of the traveling phase of the propagating light through the magnetic fluid film under external magnetic fields. It was further observed that the modulation behavior can be adjusted by using magnetic fluids of various concentrations.

\section{Acknowledgements}

This work is supported by National Science Council of ROC under Grant Nos. 93-2112-M003-007, 93-2120-M-003-001, 93-2112-M-003-017, 93-2212-E-212-001 and 93-2112-M-002-041, and by Ministry of Education, Taiwan under the Grant No. 91-N-FA01-2-4-2.

\section{References}

[1] J. Fujita, M. Levy, R.U. Ahmad, R.M. Osgood Jr., M. Randles, C. Gutierrez, R. Villareal, Appl. Phys. Lett. 75 (1999) 998.

[2] L. Yuan, L. Zhou, W. Jin, J. Yang, Opt. Lett. 27 (2002) 894.

[3] B.J. Luff, J.S. Wilkinson, J. Piehler, U. Hollenbach, J. Ingenhoff, N. Fabricuis, J. Lightwave Technol. 16 (1998) 583. 
[4] H. Porte, V. Gorel, S. Kiryenko, J.P. Goedgebuer, W. Daniau, P. Blind, J. Lightwave Technol. 17 (1999) 229 .

[5] G.V. Treyz, Electron. Lett. 27 (1991) 118.

[6] R.A. Mayer, K.H. Jung, W.D. Lee, D.L. Kwong, J.C. Campbell, Opt. Lett. 17 (1992) 1812.

[7] H. Ono, K. Misawa, K. Minoshima, A. Ueki, T. Kobayashi, J. Appl. Phys. 77 (1995) 4935.
[8] D.E. Lucchetta, R. Karapinar, A. Manni, F. Simoni, J. Appl. Phys. 91 (2002) 6060.

[9] J.W. Kang, J.J. Kim, E. Kim, Appl. Phys. Lett. 80 (2002) 1710.

[10] S.Y. Yang, Y.F. Chen, H.E. Horng, Chin-Yih Hong, W.S. Tse, H.C. Yang, Appl. Phys. Lett. 81 (2002) 4931.

[11] H.E. Horng, Chin-Yih Hong, S.Y. Yang, H.C. Yang, Appl. Phys. Lett. 82 (2003) 2434. 